$\$=19$

\title{
Secluding few lines on physiological interactions triggered by reducers
}

\author{
Muhammad Torequl Islam* \\ Northeast Biotechnology Network (RENORBIO), Postgraduate Program in Pharmaceutical Sciences, Federal University of Piauí, Teresina \\ (Piauí)-64.009-550, Brazil. \& Department of Pharmacy, Southern University Bangladesh, Mehedibag (Chittagong)-4000, Bangladesh \\ *Corresponding author E-mail: rbiotufpi.br@gmail.com
}

\begin{abstract}
Nowadays, we are very much concerned about the physiological contributions of oxidative species (e.g. - free radicals, reactive species). These include reactive oxygen/nitrogen species (ROS/RNS), vastly under continuous study in the medical concerns, emphasized on normal physiological as well as pathophysiological conditions. Being oxidizer, they have enforced us to search substances or conditions capability to counteract them, called the reducers. Doubtless, redox reaction has numerous roles in a biological system; despite we badly count the effects of ROS. This paper depicts some important interactions related to the reduction effects on the biological systems.
\end{abstract}

Keywords: Redox-Reaction; Physiological Role; Biological Interactions.

\section{Introduction}

Through the redox homeostasis cell ensures proper responses to the endogenous and exogenous stimuli. When the redox homeostasis is disturbed, oxidative stress may lead to aberrant cell death and contribute to disease development. An increase in the protonmotive force is associated with the increase in reactive oxygen species (ROS) production. A high proton-motive force slows down the flow of electrons along the respiratory chain, which in turn increases the probability of electron interaction with the molecular oxygen $\left(\mathrm{O}_{2}\right)$ and production of superoxide ion $\left(\mathrm{O}_{2}^{-}\right)$(Sena and Chandel 2012). Generally, the reduction of $\mathrm{O}_{2}$ known as a principal mechanism for ROS formation (Droge 2002) is a chain and complicated process. Under physiologic conditions, cells maintain redox balance through the generation and elimination of ROS/RNS (Kamata and Hirata 1999). A general ROS/RNS production and redox-balancing pathways has been shown in figure 1 . Reduction and the reducers also play important roles in a biological system, although we are in a usual lay a hand on ROS or oxidative effects. This paper is going to present some points, as a part of redox (reduction-oxidation) reaction, how reduction is important in physiological functions.

\section{Reducer, half of a redox system}

In general, reduction is a chemical reaction involving gaining of electrons. It always comes together with an oxidation reaction. Oxidation and reduction together are called 'redox'. A reducing agent (also called a reductant or reducer) loses (or donates) an electron to another chemical species in a redox reaction. A radical is an atom, molecule, or ion having unpaired valence electrons. Thus, it may be radical cation $\left(\mathrm{C}^{\bullet+}\right)$, radical anion $\left(\mathrm{A}^{\bullet-}\right)$ and nonionic or free radical $\left(\mathrm{F}^{\bullet}\right)$. These are mainly originated from the breaking of covalent bonds. Long lived free radicals are classified as - stable radicals, persistent radicals, and di-radicals. These are highly unstable due to their unpaired electrons and are neither oxidants nor reducing agents. However, they can impart oxidative stress. It is due to their capability to steal electron $\left(\mathrm{e}^{-}\right)$form where they can such as cellular components; these include carbohydrates, proteins, lipids and genetic materials (e.g. - DNA, RNA). In comparison to the protein and lipid molecules, a DNA molecule is more secured from oxidative stress, as it is double stranded and shielded by the histone as well as other coating proteins (Haghdoost et al. 2005).

To defend the oxidative stress, our body naturally produces antioxidants like superoxide dismutases (SOD1, -2, -3), catalase (CAT), and an assortment of peroxidase enzyme (e.g. - glutathione peroxidase $(\mathrm{GPx})$ ). Unfortunately, in severe condition, the antioxidants produced naturally by the body are not enough to neutralize all of the free radicals in a physiological system. This can be further managed by external antioxidants. Antioxidants have the remarkable ability to repair damaged molecules by donating hydrogen atoms to the molecules. Some of them also have chelating effects on free radical production, that catalyzed by heavy metals. However, the antioxidant may contain heavy metal molecules so strongly that the chemical reaction necessary to create a free radical never occurs. Thus, the activity of antioxidants is complex, as they can impart both harmful and protective roles in cells. Nonetheless, the neutralization of essential ROS/RNS may impart harmful effects on the body. Otherwise, over stimulation or suppression of the activity of body antioxidant systems are also two other harmful signals. Moreover, there is a chance of gaining dependency on external antioxidants by the physiological antioxidant systems. Certain antioxidants might increase cancer risk due to they enable cells harbour mutations to bypass senescencemediated ROS-dependent growth arrest. There are reports that oncogenic mutations can specifically increase antioxidant defense mechanisms, thus the tumour growth (DeNicola et al. 2011). In some recent evidences, reduction in SOD2, SOD isoforms, and CAT are evident to extend lifespan of the experimental animals (Van Remmen et al. 2003; Schriner et al. 2005; Yang et al. 2007). Antioxidant-mediated pro-oxidative effects are also evident (Islam 
et al. 2016a) by these days. Although, it is considered as a protective effect, but over-dose of an antioxidant may attain 'antioxidative stress'. Therefore, a balance between the oxidative stress and amount of antioxidant molecules is crucial prior to administer in our body (Islam et al. 2016b). The beneficial and harmful roles of antioxidants have been shown in Figure 2.

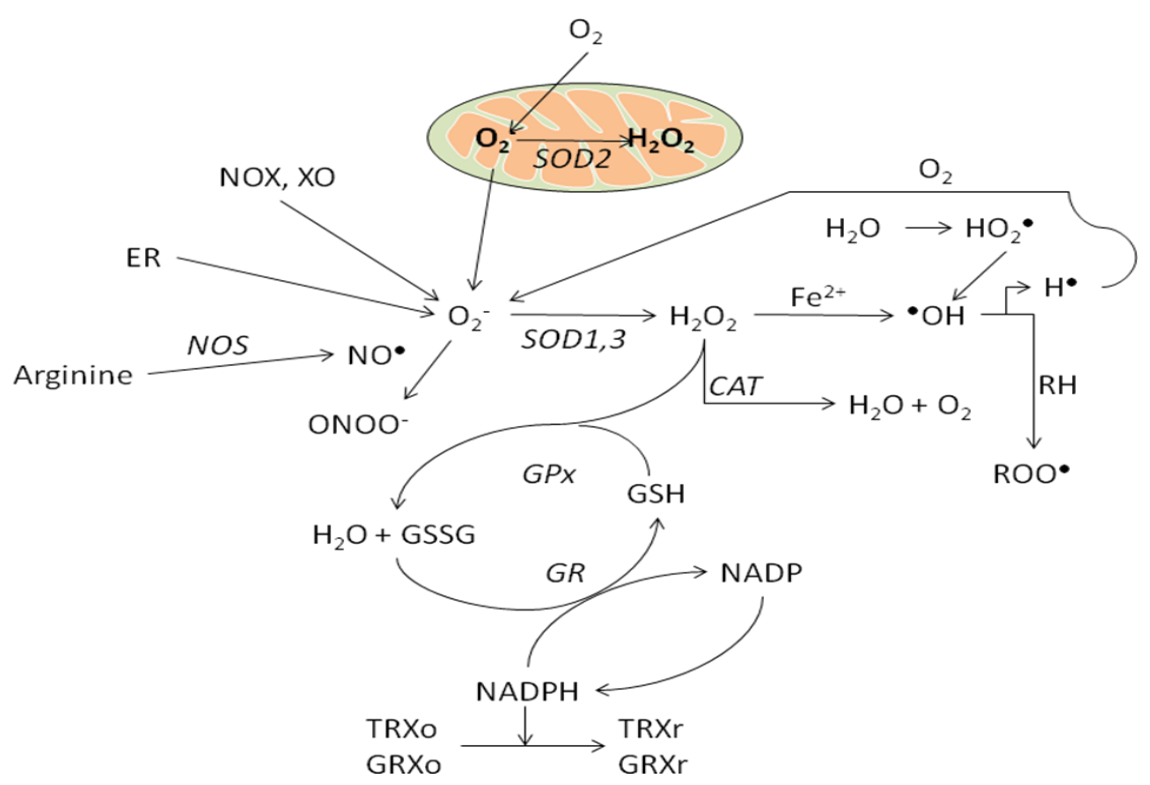

Fig. 1: Production of Reactive Species and Redox Homeostasis. [Major sites of cellular reactive species ROS/RNS generation include the mitochondrial electron transport chain, endoplasmic reticulum (ER), and the NAD (P) H oxidase (NOX) complex. Nitric oxide synthases (NOS) are the key enzymes for the production of nitric oxide (NO). Major ROS scavenging enzymes are superoxide dismutases (SOD1, 2, 3), catalase (CAT), and glutathione peroxidase (Gpx). Reduced glutathione (GSH) and nicotinamide adenine dinucleotide phosphate (NAPDH) play roles in maintaining the reduced cellular redox state. The other components in the redox system are: Glutathione reductase (GR), oxidized thioredoxin (Trxo), reduced thioredoxin (Trxr), oxidized glutaredoxin (Grxo), reduced glutaredoxin (Grxr), hydroxyl radical $(\bullet \mathrm{OH})$, peroxynitrite $\left(\mathrm{ONOO}^{-}\right)$, oxidized glutathione (GSSG) and xanthine oxidase (XO).] .

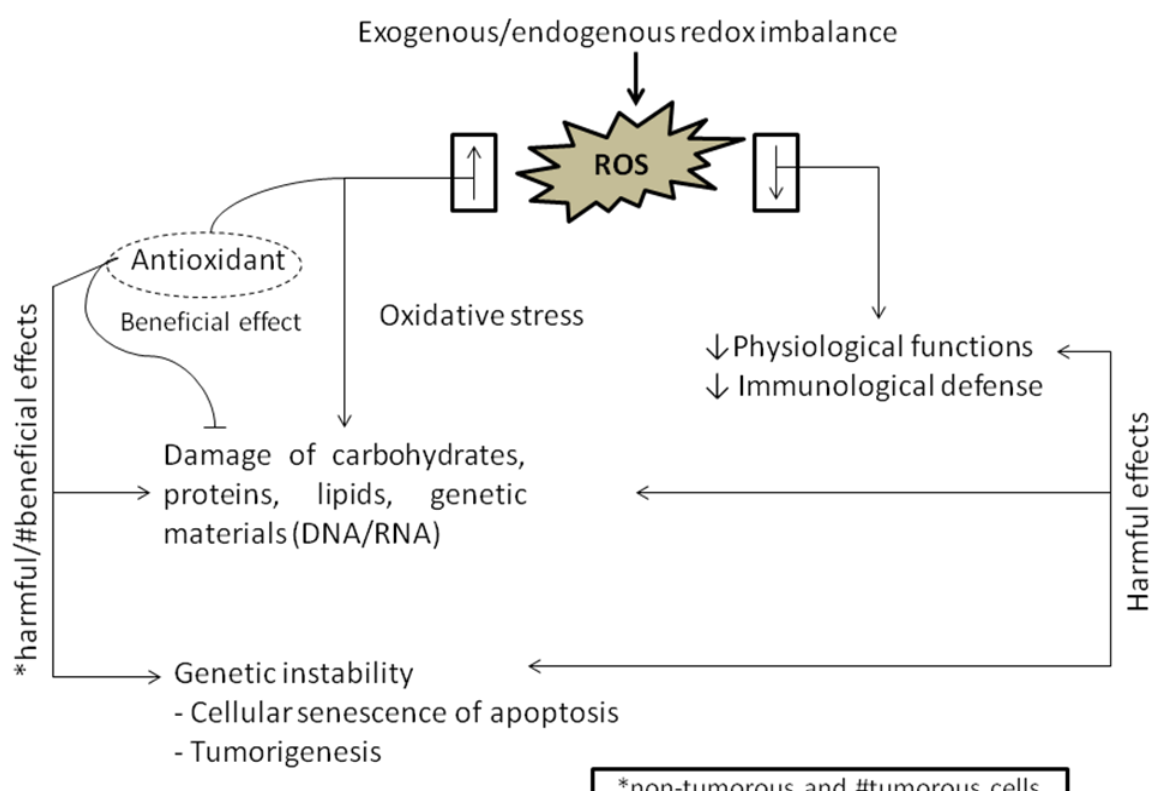

Fig. 2: Potential Beneficial/Harmful Roles on Antioxidants in Some Cellular Events.

The redox-active metals such as iron $(\mathrm{Fe})$ and copper $(\mathrm{Cu})$ can contribute ROS generation. In the presence of Fe (II) and (III), hydroxyl radical $(\bullet \mathrm{OH})$ can be generated through the Fenton reaction or Haber-Weiss reaction (Kehrer 2000). Being a cofactor of various antioxidant enzymes, reduced glutathione $(\mathrm{GSH})$ is the most abundant peptide in cells. It converts hydrogen peroxide $\left(\mathrm{H}_{2} \mathrm{O}_{2}\right)$ to molecular oxygen $\left(\mathrm{O}_{2}\right)$ through a couple of reactions (Brigelius-Flohe 2006) (Fig. 1). It is also evident to reduce a broad range of hydroperoxidases (Lee et al. 1998) and regenerate other antioxidants such as vitamin $\mathrm{C}$ and $\mathrm{E}$ to their active forms (Nakamura et al. 1997).

On the other hand, the glutaredoxin (Grx) system (also with the CXXC conserved active site) functions to reduce protein disul- fides. Grx1, Grx2, and Grx3 obtain their protein-reducing capacity of the GSH system, maintained by NADPH (Holmgren and Aslund 1995). The peroxiredoxins (Prxs) are a large family of proteins with cysteine-containing redox active centers (Rhee et al. 2005), using the peroxidatic cysteine (reactive center) to reduce hydroperoxides (Wood et al. 2003). The fatty acids, after cyclization, may form a hydroperoxide or undergo another cyclization, which produces aldehydes, including malondialdehyde (MDA) and 4-hydroxy-2-nonenal (HNE) (Pinchuk et al. 1998). MDA may react with DNA bases and results mutations in genes, while the HNE mostly reacts with the proteins and causes significant functional alterations as well as disturbs signaling pathways (Awasthi et al. 2003). 
The DNA is composed of multi-components; those are subjected to damage not only by electrophiles but also by neucleophiles. For an example, both purine and pyrimidine bases and the sugar backbone contain $\mathrm{N}$ and $\mathrm{O}$ as nucleophilic centers (Breen and Murphy 1995). Notably, a number of transcription factors contain redoxsensitive cysteine residues in their DNA-binding sites (Haddad 2002) such as nuclear factor kappa $B(N F-\kappa B)$, activator protein 1 (AP-1), hypoxic-inducible factor 1 alpha (HIF-1 $\alpha$ ) and protein 53 (p53) (Turpaev 2002). Redox-sensitive few other mechanisms include apoptosis-regulating signal kinase 1 (ASK1)-thioredoxin (TRX), c-Jun-N-terminal kinase (JNK)-glutathione-S-transferase (GST), and nuclear factor erythroid 2-related factor 2 (Nrf2)Kelch-like ECH-associated protein 1 (Keap1).

Redox reaction is also found in Met residues, in a number of amino acids, especially sulphur-containing amino acids (Drazic and winter 2014). Redox-mediated phosphorylation of I $\mathrm{KB}, \mathrm{Bcl}-2$, and p53 seems to increase the binding to their specific ubiquitin ligase $\mathrm{E} 3$ and to promote the proteosome-mediated degradation of these proteins (Bhaskar and Hay 2007).

The role of redox regulation in chromatin remodeling, which affects death/survival signals at the transcriptional level (Rahman et al. 2004). Moreover, posttranslational modifications of signaling proteins such as phosphorylation are also regulated in part through a redox-mediated mechanism (Heneberg and Draber 2005).

The peroxiredoxin (PRDX) family enzymes are the redox systems that have linked to circadian rhythms in a wide range of species (Edgar et al. 2012). The transcription factor OxyR regulates antioxidant stress response program in bacteria (Lee et al. 2004), while the forkhead box protein O (FOXO) family in mammals (Putker et al. 2013). In the nucleus, when Nrf2 binds to antioxidant response elements containing within the promoters of antioxidant genes, including those encoding GST and haem oxygenase 1 (HO1), augments the overall defense of the cell against ROS (Taguchi et al. 2011). The redox-dependent inactivation of ATG4 is also evident to increase the formation of autophagosome, mainly during starvation (Scherz-Shouval et al. 2007). A change in redox-dependent regulation of the transcription factor nuclear factor of activated T cells 1 (NfaT1) can decrease in mitochondrial ROS (mROS) production, thus the defects in $\mathrm{T}$ cell activation and clonal expansion (Zhang et al. 2013). Redox homeostasis is also seen with polycomb protein BMI1, which is required for stem cell self-renewal (Liu et al. 2009; Chatoo et al. 2009) and in genomic stability (Li et al. 2010).

\section{Conclusion}

Our cells are rich with both, oxidizing and reducing mediators Being a part of a complete chemical reaction, reduction is always important in physiological functions. Therefore, not only the oxidizers' but also reducers' effects we count. The ultimate effects are linked to the sites, situations, and levels; that are concluding messages to the physiologists, medicinal scientists as well as therapists.

\section{Conflict of interest}

None declared.

\section{References}

[1] Sena LA, Chandel NS. Physiological roles of mitochondrial reactive oxygen species. Mol Cell 2012; 48:158-167. https://doi.org/10.1016/j.molcel.2012.09.025.

[2] Droge W. Free radicals in the physiological control of cell function Physiol Rev 2002; https://doi.org/10.1152/physrev.00018.2001.

[3] Kamata H, Hirata H. Redox regulation of cellular signalling. Cell Signal 1999; 11:1-14. https://doi.org/10.1016/S08986568(98)00037-0
[4] Haghdoost S, Czene S, Naslund I, Skog S, Harms-Ringdahl M. Extracellular 8-oxo-dG as a sensitive parameter for oxidative stress in vivo and in vitro. Free Radic Res 2005; 39:153-162. https://doi.org/10.1080/10715760500043132.

[5] DeNicola GM. Karreth FA, Humpton TJ, Gopinathan A, Wei C, Frese K, et al. Oncogene-induced Nrf2 transcription promotes ROS detoxification and tumorigenesis. Nature 2011; 475:106-109. https://doi.org/10.1038/nature10189.

[6] Van Remmen H, Ikeno Y, Hamilton M, Pahlavani M, Wolf N, Thorpe SR, et al. Life-long reduction in MnSOD activity results in increased DNA damage and higher incidence of cancer but does not accelerate aging. Physiol Genom 2003; 16:29-37. https://doi.org/10.1152/physiolgenomics.00122.2003.

[7] Schriner SE, Linford NJ, Martin GM, Treuting P, Ogburn CE, Emond M, et al. Extension of murine life span by overexpression of catalase targeted to mitochondria. Sci 2005; 308:1909-1911. https://doi.org/10.1126/science.1106653.

[8] Yang W, Li J, Hekimi SA. Measurable increase in oxidative damage due to reduction in superoxide detoxification fails to shorten the life span of long-lived mitochondrial mutants of Caenorhabditis elegans. Genetics 2007; 177:2063-2074. https://doi.org/10.1534/genetics.107.080788.

[9] Islam MT, Streck L, Paz MFCJ, Sousa JMC, Alencar MVOB, Mata AMOF, et al. Preparation of phytol-loaded nanoemulsion and screening for antioxidant capacity. Int Arch Med 2016; 9:1-15. https://doi.org/10.3823/1941.

[10] Islam MT, Ali ES, Sousa JMC, Santos JVO, Paz MFCJ, Lima RMT, et al. Physiological contributions of reactive oxygen species. Photon eBooks. Ed. I, Nov 2016. Pages 1-13. UBN: 015A94510112030.

[11] Kehrer JP. The Haber-Weiss reaction and mechanisms of toxicity. Toxicol 2000; 149:43-50. https://doi.org/10.1016/S0300483X(00)00231-6.

[12] Brigelius-Flohe R. Glutathione peroxidases and redox-regulated transcription factors. Biol Chem 2006; 387:1329-1335 https://doi.org/10.1515/BC.2006.166.

[13] Lee SR, Kwon KS, Kim SR, Rhee SG. Reversible inactivation of protein-tyrosine phosphatase 1B in A431 cells stimulated with epidermal growth factor. J Biol Chem 1998; 273:15366-15372. https://doi.org/10.1074/jbc.273.25.15366.

[14] Nakamura H, Nakamura K, Yodoi J. Redox regulation of cellular activation. Annu Rev Immunol 1997; 15:351-369. https://doi.org/10.1146/annurev.immunol.15.1.351.

[15] Holmgren A, Aslund F. Glutaredoxin. Methods Enzymol 1995; 252:283-292. https://doi.org/10.1016/0076-6879(95)52031-7.

[16] Rhee SG, Yang K-S, Kang SW, Woo HA, Chang T-S. Controlled elimination of intracellular $\mathrm{H}_{2} \mathrm{O}_{2}$ : regulation of peroxiredoxin, catalase, and glutathione peroxidase via post-translational modification. Antioxid Redox Signal 2005; 7:619-626. https://doi.org/10.1089/ars.2005.7.619.

[17] Wood ZA, Schroder E, Robin Harris J, Poole LB. Structure, mechanism and regulation of peroxiredoxins. Trends Biochem Sci 2003; 28:32-40. https://doi.org/10.1016/S0968-0004(02)00003-8.

[18] Pinchuk I, Schnitzer E, Lichtenberg D. Kinetic analysis of copperinduced peroxidation of LDL. Biochim Biophys Acta 1998; 1389:155-172. https://doi.org/10.1016/S0005-2760(97)00139-2.

[19] Awasthi YC, Sharma R, Cheng JZ, Yang Y, Sharma A, Singhal SS, et al. Role of 4-hydroxynonenal in stress-mediated apoptosis signaling. Mol Aspects Med 2003; 24:219-230 https://doi.org/10.1016/S0098-2997(03)00017-7.

[20] Breen AP, Murphy JA. Reactions of oxyl radicals with DNA. Free Radic Biol Med 1995; 18:1033-1077. https://doi.org/10.1016/08915849(94)00209-3.

[21] Haddad JJ. Antioxidant and prooxidant mechanisms in the regulation of redox $(\mathrm{y})$-sensitive transcription factors. Cell Signal 2002; 14:879-897. https://doi.org/10.1016/S0898-6568(02)00053-0.

[22] Turpaev KT. Reactive oxygen species and regulation of gene expression. Biochemistry (Moscow) 2002; 67:281-292. https://doi.org/10.1023/A:1014819832003.

[23] Drazic A, Winter J. The physiological role of reversible methionine oxidation. Biochim Biophys Acta 2014; 1844:1367-1382. https://doi.org/10.1016/j.bbapap.2014.01.001.

[24] Bhaskar PT, Hay N. The two TORCs and Akt. Dev Cell 2007; 12:487-502. https://doi.org/10.1016/j.devcel.2007.03.020.

[25] Rahman I, Marwick J, Kirkham P. Redox modulation of chromatin remodeling: impact on histone acetylation and deacetylation, $\mathrm{NF}$ kappaB and pro-inflammatory gene expression. Biochem Pharmacol 2004; 68:1255-1267. https://doi.org/10.1016/j.bcp.2004.05.042.

[26] Heneberg P, Draber P. Regulation of cys-based protein tyrosine phosphatases via reactive oxygen and nitrogen species in mast cells 
and basophils. Curr Med Chem 2005; 12:1859-1871. https://doi.org/10.2174/0929867054546636.

[27] Edgar RS, Green EW, Zhao Y, van Ooijen G, Olmedo M, Qin X, et al. Peroxiredoxins are conserved markers of circadian rhythms. Nature 2012; 485:459-464. https://doi.org/10.1038/nature11088.

[28] Lee C, Lee SM, Mukhopadhyay P, Kim SJ, Lee SC, Ahn WS, et al. Redox regulation of OxyR requires specific disulfide bond formation involving a rapid kinetic reaction path. Nature Struct Mol Biol 2004; 11:1179-1185. https://doi.org/10.1038/nsmb856.

[29] Putker M, Madl T, Vos HR, de Ruiter H, Visscher M, van den Berg $\mathrm{MC}$, et al. Redox-dependent control of FOXO/ DAF 16 by transportin $1 . \quad$ Mol Cell 2013; 49:730-742. https://doi.org/10.1016/j.molcel.2012.12.014.

[30] Taguchi K, Motohashi H, Yamamoto M. Molecular mechanisms of the Keap1-Nrf2 pathway in stress response and cancer evolution. Genes Cells 2011; 16:123-140. https://doi.org/10.1111/j.13652443.2010.01473.x.

[31] Scherz-Shouval R, Shvets E, Fass E, Shorer H, Gil L, Elazar Z. Reactive oxygen species are essential for autophagy and specifically regulate the activity of Atg4. EMBO J 2007; 26:1749-1760. https://doi.org/10.1038/sj.emboj.7601623.

[32] Zhang Y, Choksi S, Chen K, Pobezinskaya Y, Linnoila I, Liu ZG ROS play a critical role in the differentiation of alternatively activated macrophages and the occurrence of tumor-associated macrophages. Cell Res 2013; 23:898-914. https://doi.org/10.1038/cr.2013.75.

[33] Liu J, Cao L, Chen J, Song S, Lee IH, Quijano C, et al. Bmil regulates mitochondrial function and the DNA damage response pathway. Nature 2009; 459:387-392. https://doi.org/10.1038/nature08040.

[34] Chatoo W, Abdouh M, David J, Champagne M-P, Ferreira J, Rodier F, et al. The polycomb group gene Bmil regulates antioxidant defenses in neurons by repressing p53 pro-oxidant activity. J Neurosci 2009; 29:529-542. https://doi.org/10.1523/JNEUROSCI.5303-08.2009.

[35] Li TS, Marban E. Physiological levels of reactive oxygen species are required to maintain genomic stability in stem cells. Stem Cells 2010; 28:1178-1185. https://doi.org/10.1002/stem.438. 